\title{
Effect of Myo-inositol and N-acetyl-L-cysteine on processed human spermatozoa for use in modern methods of fertility treatment
}

\author{
Maryam Nemati $^{(\mathbb{1}}$, Sadra Ansaripour ${ }^{\mathbb{D}}$, Naghme Samadi $^{*}{ }^{(\mathbb{D}}$ \\ ${ }^{1}$ Assistant Professor, Department of Gynecology and Obstetrics, Shahrekord University of Medical Sciences, Shahrekord, Iran. \\ ${ }^{2}$ Medical Student, Student Research Committee, Shahrekord University of Medical Sciences, Shahrekord, Iran.
}

*Corresponding Author: Naghme Samadi; Student Research Committee, Shahrekord University of Medical Sciences, Shahrekord, Iran. Tel: +989132830164; Email: naghme.samadi.92@gmail.com

\begin{abstract}
Background and aims: Today, the prevalence of structural and functional disorders of sperm and subsequently the possibility of occurrence of autism, spontaneous abortion, and infertility has increased dramatically. This study aimed to investigate the potential effects of myoinositol and $\mathrm{N}$-acetyl-L-cysteine (NAC) on the improvement of processed spermatozoa indices.

Methods: In this study, 63 patients with male factor infertility were studied in Hazrat Zahra Infertility Center of Shahrekord. To do liquefaction, the semen sample was incubated at $37^{\circ} \mathrm{C}$ for 20 minutes. The Density Gradient Centrifugation protocol was used to process sperm. The processed sperm samples were incubated at $37^{\circ} \mathrm{C}$ for 20 minutes in four groups of control, myo-inositol $(2 \mathrm{mg} / \mathrm{mL}), \mathrm{NAC}$ antioxidant $(10 \mathrm{Mm} / \mathrm{L})$ and myo-inositol+NAC. All biological parameters of sperm were evaluated using Makler slide, SQA-V sperm analyzer gold version 2.48 , TUNEL assay, and Toluidine blue staining.

Results: In the present study, 63 men aged 22-58 years with infertility complaints were studied. The NAC group and the myo-inositol group exhibited significantly higher motility in comparison with the control group $(P<0.001)$. The NAC group also had a significantly higher number of TUNEL-negative cells and a lower number of TUNEL-positive cells than the control group $(P<0.01)$, but in other variables, the differences were not statistically significant. The morphology, S0, S1, S2 and staining types in the intervention groups did not show any significant difference compared to those in the control group $(P>0.05)$.

Conclusion: NAC and myo-inositol were able to improve certain indices such as sperm motility and integrity; therefore, the quality of sperm could be improved by these drugs to treat infertility.

Keywords: Myo-inositol, N-acetyl-L-cysteine, Infertility treatment, Biological parameters of sperm, IVF, ICSI
\end{abstract}

Received: 16 July 2019, Accepted: 16 September 2019, ePublished: 29 April 2020

\section{Introduction}

Today, infertility has become one of the main concerns of the community, especially medical circles (1). According to the statistics provided by Avicenna Research Institute (obtained from a study on 17000 couples over a 2-year period), the prevalence of infertility in Iran is estimated to be $20.2 \%$ (almost twice the global average declared by the $\mathrm{WHO}$ ). Evidence has revealed that $40 \%$ of the cases of infertility are due to male factors, $40 \%$ are due to female factors, $10 \%$ are due to both and $10 \%$ of infertility cases are due to unknown causes (1). Although the molecular basis of idiopathic infertility remains to be elucidated, it seems that high level of reactive oxygen species (ROS) in semen plasma is one of the most important causes of idiopathic infertility. It should be noted that low and normal concentrations of ROS in semen plasma (hydrogen peroxidase or free radicals such as nitric and anion peroxidase) stimulate and improve sperm capacitation process, increase sperm activity, induce acrosome reaction, increase the sperm ability to bind to the zona pellucida and ultimately lead to fertility $(2,3)$. At sperm capacitation, high levels of ROS and high concentrations of intracellular calcium and tyrosine kinase lead to an increase in cAMP and consequently improve sperm motility $(2,4)$. High concentrations of ROS disrupt the structure of DNA, eliminate the integrity of the DNA structure, reduce the sperm motility, disrupt the integrity of the cytoplasmic membrane structure of the sperm and cause a reduction in the fertility potential of the sperm. Regarding the existence of a direct and significant relationship between the deficiency of antioxidants and infertility due to male factors, the use of some antioxidants such as $\mathrm{N}$-acetylL-cysteine (NAC), coQ10, L-carnitine, zinc, selenium, and vitamins $\mathrm{C}$ and $\mathrm{E}$, which reduce ROS and increase

(C) 2020 The Author(s); Published by Shahrekord University of Medical Sciences. This is an open-access article distributed under the terms of the Creative Commons Attribution License (http://creativecommons.org/licenses/by/4.0), which permits unrestricted use, distribution, and reproduction in any medium, provided the original work is properly cited. 
fertility, is recommended for the treatment of male factor infertility (3).

NAC is one of the most powerful antioxidants with positive effects on fertility. This compound can directly scavenge the free radicals of oxygen and cause the survival of spermatogonia germ cells in the seminiferous tubules, an increase in sperm motility, a significant decrease in ROS levels in semen plasma, an increase in sperm chromatin density, and a decrease in sperm fragmentation $(4,5)$. NAC has been shown to reduce the cytoplasmic reticulum stress by inhibiting ROS in Sertoli cells. This compound reduces lipid peroxidation, preserves the cell structure, especially Sertoli cells, and maintains their function (6).

Infertility treatment studies have shown the role of oral myo-inositol in morphogenesis, lipid synthesis, cell cytogenesis, and biological characteristics of sperm and fertility $(4,7)$. Myo-inositol is one of the nine isomers of inositol and belongs to group B vitamins. Myo-inositol is one of the synthesis precursors of phosphatidylinositol polyphosphate, and as a second messenger precursor, plays an important role in regulating intracellular calcium levels, regulating sperm motility, increasing sperm capacitation, maintaining mitochondrial membrane potential, integrating the cellular skeletal system, regulating gene expression, regulating the lipid content of sperm cytoplasmic membrane, and producing acrosome reaction (2,8-10). Studies have shown that myo-inositol plays a crucial role in sperm maturation and migration of cells from the epididymis and its concentration is higher in the seminiferous tubule fluids than in semen plasma $(11,12)$. It also plays an important role in chemotaxis including human sperm chemotaxis through the activation of phospholipase $\mathrm{C}$, which leads to the production of InsP3, opening of calcium channels, and increase of intracellular calcium concentrations in sperm flagellum (12). This drug is commonly used for the treatment of male factor infertility, with promising results reported on the improvement of semen quality by using it.

Given the increasing prevalence of male factor infertility, the increase in the need for modern methods of infertility treatment, efforts to increase the quality of sperm processed for intrauterine insemination (IUI) or in vitro fertilization/intracytoplasmic sperm injection (IVF/ICSI), and the necessity of using drug compounds to improve sperm fertility indexes and chromatin health, this study was done to investigate the effect of myo-inositol $(2 \mathrm{mg} /$ $\mathrm{mL}), \mathrm{NAC}(10 \mathrm{mmol} / \mathrm{L})$ and NAC + myo-inositol $(2 \mathrm{mg} /$ $\mathrm{mL}, 10 \mathrm{mmol} / \mathrm{L}$ ) on sperm fertility. Since sperm selection according to normal biological parameters cannot indicate the sperm DNA integrity (13) and there is no correlation between the biological parameters of sperm and the severity of DNA damage (14), the effect of these compounds on the rate of DNA damage and chromosomal health of the sperm was also examined.

\section{Materials and Methods}

This clinical trial was conducted during 2018-2019 at Shahrekord University of Medical Sciences and its protocol was approved by the Deputy of Research and Technology of the University and registered in the Iranian Registry of Clinical Trials (identifier: IRCT20190204042618N2).

To select participants, convenience sampling was done in male patients referring to the Infertility Center in Shahrekord. Assuming that during the study, each qualitative parameter of the sperm changed by at least half of the standard deviation, the sample size was determined to be at least 63 individuals based on a confidence level of $95 \%$ and power of $80 \%$ (8) (It should be noted that the samples obtained from these individuals are divided into 4 sections after processing).

In this study, 63 patients with male factor infertility, the history of infertility for at least 12 months, spermatozoa count of less than $106 \times 15 / \mathrm{mL}$, progressive motility of less than $32 \%$, and normal morphology of less than $4 \%$ were included. Additionally, patients who consumed alcohol, minerals, multivitamin or any fertility enhancer in the last three months or used unauthorized compounds and drugs were excluded.

Patients' characteristics such as age, weight, body mass index, occupation, marriage duration, years of infertility, smoking habits, alcohol and drug use, physical activity, chemical exposure, and history of disease or surgery were recorded. Before participating in the study, the patients signed the consent form. The samples were recorded anonymously and the samples' specifications were determined only by a computer code. After semen samples were received under standard conditions in sterile plastic containers and were sent to the laboratory, they were incubated at $37^{\circ} \mathrm{C}$ for 20 minutes for liquefaction.

The Density Gradient Centrifugation protocol was used to process sperm. After sperm preparation by this technique, all of the biological parameters of the sperm (viability of sperm, normal morphology, and total motile count) were evaluated using Makler slides (phase contrast microscope) and SQA-V sperm analyzer gold version 2.48. Then, the processed sperm sample was divided into four parts as follows:

1) Control group: One part of the sample with no exposure to drugs was incubated for 20 minutes at $37^{\circ} \mathrm{C}$.

2) Treatment group 1: One part of the sample was incubated with $2 \mathrm{mg} / \mathrm{mL}$ of myo-inositol at $37^{\circ} \mathrm{C}$ for 20 minutes.

3) Treatment group 2: One part was exposed to the NAC antioxidant $(10 \mathrm{mM} / \mathrm{L})$ and incubated at $37^{\circ} \mathrm{C}$ for 20 minutes (16).

4) Treatment group 3: The last portion of the sperm sample was exposed to myo-inositol $(2 \mathrm{mg} / \mathrm{mL})+\mathrm{NAC}$ $(10 \mathrm{mM} / \mathrm{L})$ and incubated at $37^{\circ} \mathrm{C}$ for 20 minutes under the same conditions as the previous ones. 
After the completion of the incubation period in all groups, the biological parameters of the sperm were evaluated and recorded by an andrology specialist.

\section{Calculating total motile sperm count}

Using a macroscopic technique, a volume of $10 \mu \mathrm{L}$ of semen sample was left in a counting chamber at room temperature. Immediately after placing the sample in the chamber and preparing it, the number and motility of the sperm were determined and the number of motile and immotile spermatozoa was reported as a percentage.

\section{Papanicolaou staining}

To evaluate the specific characteristics of cell structure and sperm morphology and accurate examination of the sperm head, middle piece and tail defects, a thin smear was prepared from the samples of the control and treatment groups and then evaluated by Papanicolaou staining. In this method, after washing the semen sample with AllGrad Wash (three times, each time for 5 minutes) and preparing smear, hematoxylin was applied to the samples for 3 minutes. Then, the slides were dehydrated and stained with OG. Ultimately, after rehydrating the slides with different concentrations of alcohol, we used 50A12 stain, and after drying and mounting the slides, evaluations were performed at $40 \mathrm{X}$ and $100 \mathrm{X}$ magnifications. In this study, head defects (acrosome structures), neck defects (structure of the sperm neck and cytoplasmic droplets), and tail defects (coiled, double, and short tails) were investigated.

\section{Toluidine blue staining}

Similarly, to evaluate the chromosomal structure of the sperm, a thin smear was prepared from different groups and stained with Toluidine blue. In this method, 96\% ethanol acetone fixation was used (1:1 ratio), and after drying the slides, $\mathrm{HCl}$ was used for 5 minutes at $4^{\circ} \mathrm{C}$ to conduct hydrolysis. Then, the slides were washed several times by washing medium and admixed with Toluidine blue at a concentration of $1 \%$ and $\mathrm{pH} 3.5$. In order to make the smears transparent, they were placed in a jar containing xylene at room temperature for 5 minutes. Finally, after mounting the slides, they were evaluated at 40X and 100X magnifications. The indexes measured in this staining method include $S_{0}, S_{1}$ and $S_{2}$, which are defined as follows:

- $S_{0}$ contains healthy and intact spermatozoa that appear in bright blue or bright purple.

- $S_{1}$ contains unhealthy spermatozoa that can appear semi-colored.
- $\mathrm{S}_{2}$ contains abnormal spermatozoa that appear in extremely dark purple.

\section{TUNEL assay}

In TUNEL assay, a thin smear of unprocessed (control) and processed (target groups) sperm cells was prepared and fixed with paraformaldehyde for one hour. After placing the prepared smears in the permeable solution and washing them with phosphate buffer saline (PBS), staining was carried out according to the instructions of the TUNEL assay kit (Roche, 11684817910). The evaluation of the prepared slides and examinations of positivity and negativity of the cells were performed using a fluorescence microscope at $510 \mathrm{~nm}$.

Sperm with a normal chromosomal structure and undamaged and dense chromatin appeared in green, whereas sperm with damaged DNA and chromosome appeared in yellowish green color. In each slide, 100 sperms were counted and the percentages of normal and abnormal sperms were calculated.

\section{Statistical analysis}

The data were entered into the SPSS and GraphPad software. The normally distributed quantitative variables were expressed as mean $\pm \mathrm{SD}$, and non-normally distributed quantitative variables were expressed as the median with interquartile range. Additionally, qualitative variables were expressed as frequency and percentage. The comparison of variables in groups was performed by Friedman test followed by Dunn's test. $P<0.05$ was considered significance level.

\section{Results}

In this study, a total of 63 male individuals aged 22 to 58 years were enrolled. The infertility period, weight, height, and body mass index of patients are shown in Table 1.

The rates of motility and normal morphology of sperm in the studied groups are shown in Table 2. Motility rate in the control group significantly changed after sperm processing according to the results of Density Gradient Centrifugation $(P<0.001)$. It should be noted that sperm

Table 1. Characteristics of the patients studied

\begin{tabular}{lccc}
\hline Variable & Minimum & Maximum & Mean \pm SD \\
\hline Age $(\mathrm{y})$ & 22 & 58 & $7.2 \pm 35.1$ \\
Infertility period $(\mathrm{y})$ & 1 & 27 & $5.75 \pm 6.43$ \\
Weight $(\mathrm{kg})$ & 58 & 110 & $9.9 \pm 78.1$ \\
Height $(\mathrm{cm})$ & 165 & 189 & $5.34 \pm 177.02$ \\
Body mass index & 19.3 & 34.3 & $2.56 \pm 24.9$ \\
\hline
\end{tabular}

Table 2. Motility and morphology of patients

\begin{tabular}{lcccc}
\hline \multirow{2}{*}{ Variable } & \multirow{2}{*}{ Before intervention } & \multicolumn{3}{c}{ Studied groups } \\
\cline { 3 - 5 } & $30.8(26-35)$ & Control & NAC & Myo \\
\hline Motility & $2.5(2.1-3)$ & $65(35-50)$ & $50(40-55)$ & $47(40-55)$ \\
Morphology & $6(5-7)$ & $6(5.1-7)$ & $6(5-7)$ & $5.9(5-6.9)$ \\
\hline
\end{tabular}

Data are presented as median (interquartile range). 
with better motility and morphology are separated by Density Gradient Centrifugation. In the NAC group, motility showed a significant difference compared to the control group $(P<0.001)$. The myo-inositol group showed a significant difference in motility compared to the control group $(P<0.001)$. Normal morphology rate was significantly higher in the control group after sperm processing according to the Density Gradient Centrifugation results $(P<0.001)$, but control, NAC, Myo-inositol, and NAC + Myo-inositol groups did not significantly differ in this variable $(P=0.063)$ (Figure 1$)$.

According to the results of toluidine blue staining and chromatin density evaluation, there was no significant difference in $S_{0}, S_{1}$, and $S_{2}$ between the experimental groups $(P=0.082)$ (Figures 2 and 3$)$.

According to the results of TUNEL assay, there was a significant difference between the NAC and the control group, indicating that the number of sperm cells that had negative TUNEL staining was higher in the NAC group $(P=0.004)$ and conversely, the number of TUNELpositive cells was significantly lower in this group than in the control group $(P=0.004)$. There was no significant difference between the other groups $(P=0.281)$ (Figure 4$)$.

In testing viability by Trypan blue staining based on the membrane responsiveness of the cells to the permeable staining materials, there was no significant difference

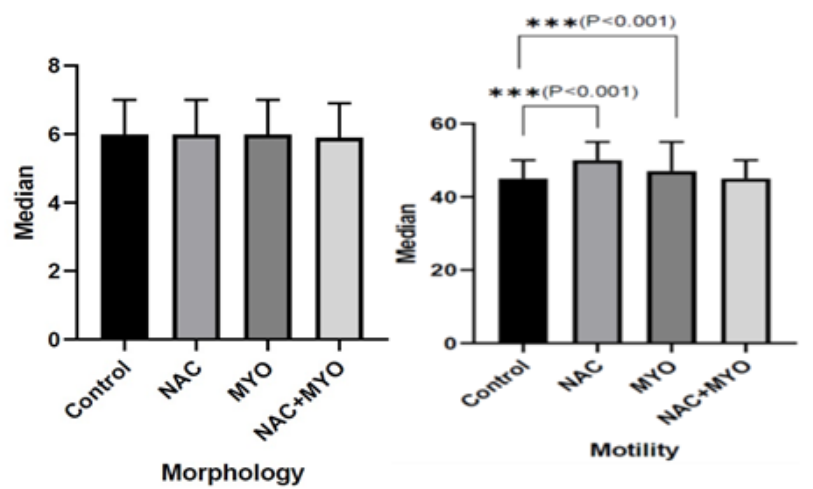

Figure 1. Normal Morphology and Motility Rate in the Studied Groups.

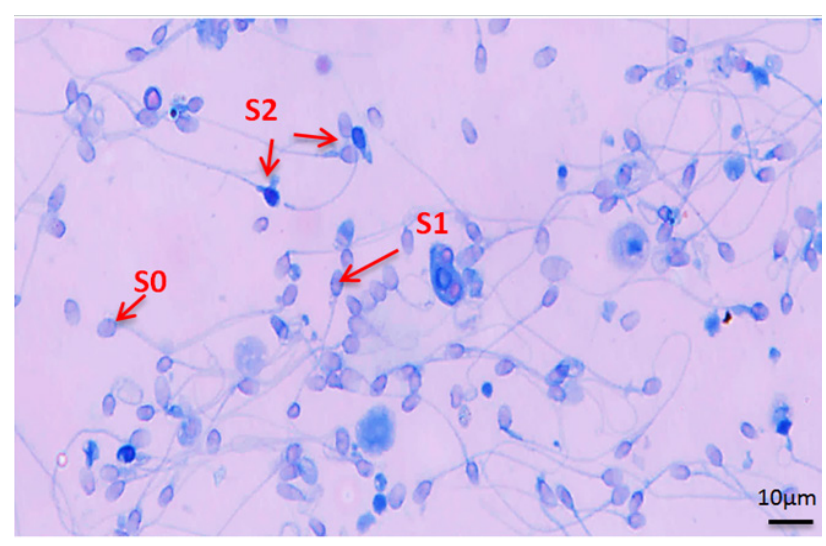

Figure 2. Toluidine blue staining and $\mathrm{S}_{0^{\prime}} \mathrm{S}_{1}$, and $\mathrm{S}_{2}$ sperms.
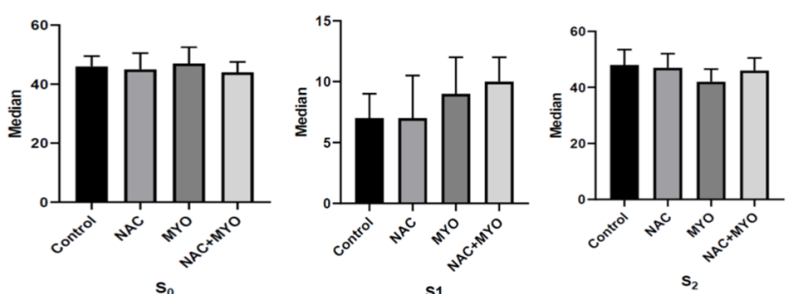

Figure 3. $\mathrm{S}_{0}, \mathrm{~S}_{1}$ and $\mathrm{S}_{2}$ in the studied groups.
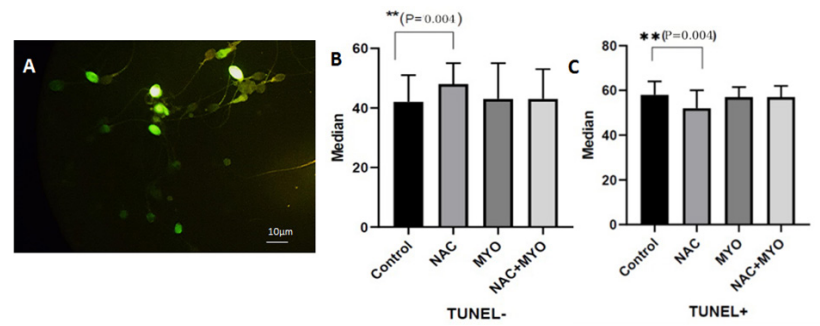

Figure 4. TUNEL assay. (A) The Bright Green Cells Show the TUNEL-positive Cells with Damaged Chromatin; (B) Percentage of TUNEL-negative Cells; and (C) Percentage of TUNEL-positive Cells in the Studied Groups

between the experimental groups $(P=0.312)$ (Figure 5).

After Papanicolaou staining, the control group did not show any significant difference in sperm head morphology (including normal, big and small head) and sperm tail morphology (including coil, double and short tail), and cytoplasmic droplet after sperm processing according to the results of density gradient centrifugation. There was also no significant difference between the experimental groups $(P=0.159)$ (Figures 6 and 7$)$.

\section{Discussion}

In the present study, the effect of myo-inositol and NAC and their combination on the improvement of the quality and quantity of the processed spermatozoa for use in modern methods of fertility treatment (IVF/ICSI) was evaluated at Hazrat Zahra Infertility Center of Shahrekord. For this purpose, a total of 63 men aged 22 to 58 years were enrolled in the study.

In this study, the NAC and myo-inositol group showed significantly higher motility than the control group. The number of TUNEL-negative cells was also significantly higher in the NAC group compared to the control group and the number of TUNEL-positive cells was significantly lower in the NAC group than in the control group, but other groups did not show any significant difference in these two factors. The rate of normal morphology, $S_{0}, S_{1}$, and $S_{2}$ as well as the results of different staining methods in intervention groups did not show any significant difference compared to those in the control group.

In the study by Comhaire et al, the effect of oral antioxidants (3-month treatment with NAC $600 \mathrm{mg}$ and vitamins $\mathrm{A}+\mathrm{E}$ ) and essential fatty acids was studied on sperm biology in infertile men. In that study, the 
improvement of sperm parameters, decrease in ROS and 8-OH-Dg (oxidized DNA of the sperm), increase in acrosome reaction and spermatozoa membrane fluidity after drug administration were not significant. The total number of sperm increased from 7.4 million to 12.5 million. The pregnancy rate of patients who used the sperm exposed to these compounds reached $4.5 \%$. In this study, the motility and normal morphology rate of sperm did not change significantly (15). The results of this study are consistent with the present study because NAC did not affect morphology, although in the present study, in vitro exposure to these drugs lasted only 20 minutes, which is different from exposure through oral and long-term administration.

Oeda et al used NAC to reduce ROS in human semen samples. The amount of ROS in semen plasma decreased
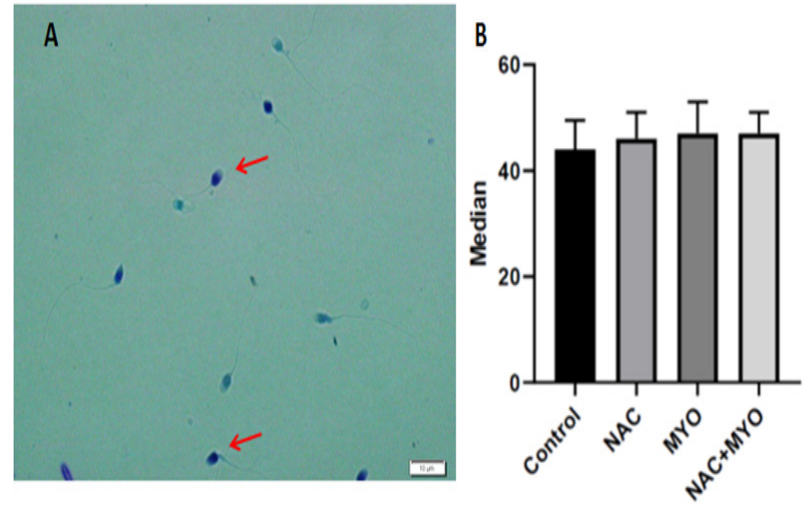

Viability

Figure 5. (A) Trypan Blue Staining. Arrows show the penetration of stain into the sperm cells and reduction of their viability; (B) Viability Rate in Different Groups. significantly after the use of medication for 20 minutes. In addition, in this study, the total sperm motility significantly increased. The researchers recommended using NAC antioxidants to improve sperm function (16). The results of the present study on the increase of sperm motility in the NAC and myo-inositol groups are consistent with the results of the cited study (16).

Paradiso Galatioto et al observed a significant increase in spermatozoa count (up to 20 times) after oral treatment with $600 \mathrm{mg}$ NAC and antioxidants (along with various vitamins and minerals). Treatment with antioxidants had no significant effect on total sperm motility, normal morphology, and rate of pregnancy (17). Condorelli et al studied the effect of myo-inositol on spermatozoa apoptosis and mitochondrial function of sperm in patients with oligo-terato-asthenozoospermia and reported that

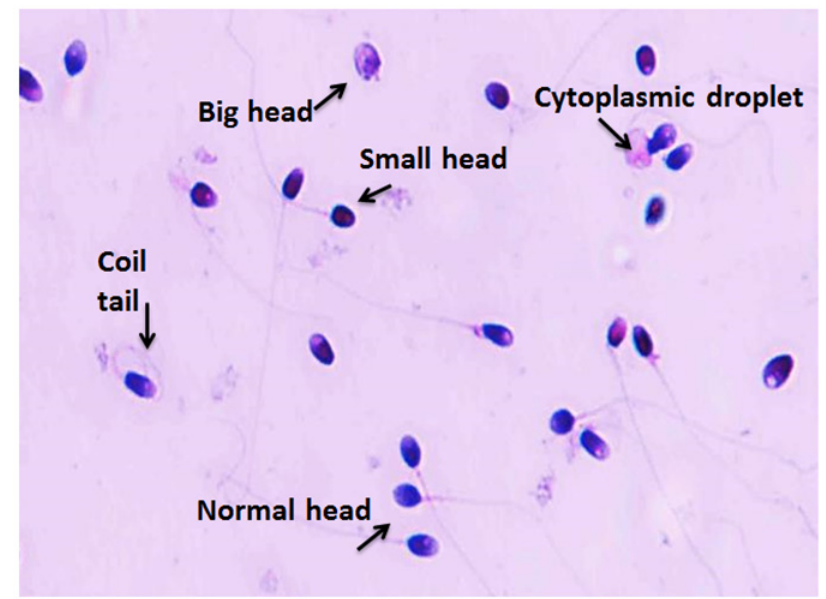

Figure 6. Papanicolaou staining for the evaluation of sperm morphology.
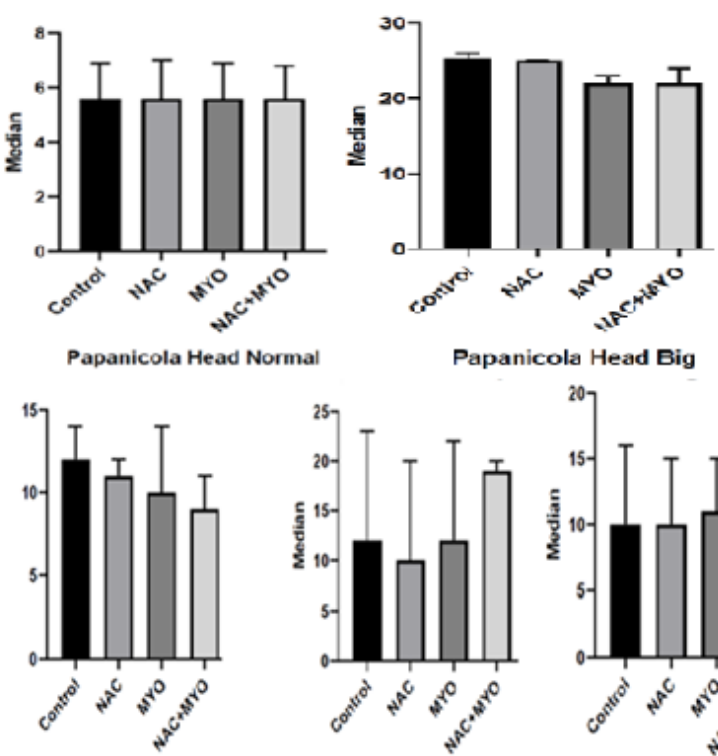

Double tail

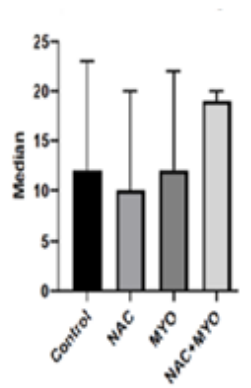

coil tail

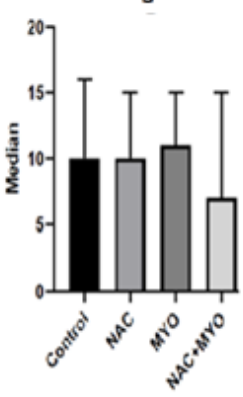

Papanicola short tail

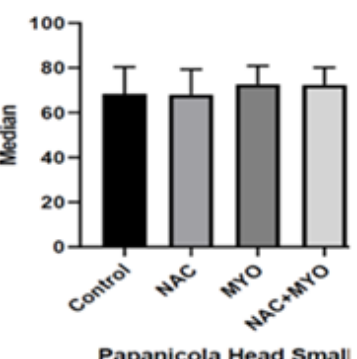

Papanicola Head Smal

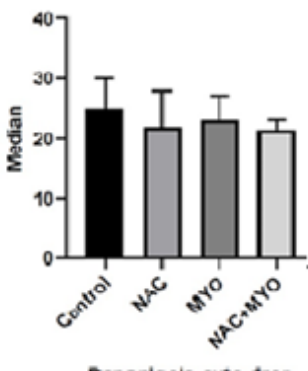

Papanicola cyto drop

Figure 7. Evaluation of sperm morphology using papanicolaou staining . 
the drug had no effect on the improvement of sperm mitochondria and its function. In this study, myo-inositol significantly increased the number of spermatozoa with high mitochondrial membrane potential. The number of spermatozoa with low mitochondrial membrane potential in patients with oligo-terato-asthenozoospermia also significantly reduced. Additionally, the drug had no significant effect on sperm chromatin density (18), which is consistent with our study.

Unfortunately, the prevalence of infertility in the world, especially in Iran, is increasing. According to the latest epidemiological study, the prevalence of infertility in Iran is much higher than global statistics and is estimated to be $20.2 \%$. Approximately $40 \%$ of infertility is due to male factors, the most important of which are spermatic (oligospermia, teratospermia, asthenospermia, and azoospermia), anatomical, and hormonal disorders. Therefore, given the increasing prevalence of infertility in the male population of the country, the need for male fertility treatment has become one of the main priorities of all infertility treatment centers. The selection of a sufficient number of spermatozoa with normal biological parameters and the health of the genomic and chromosomal structure can have a direct and significant effect on the success rate of fertility treatments, fertilization rate, cleavage rate, number of fetuses, quality of the fetus, and ultimately pregnancy rate. Therefore, selecting the protocol for sperm preparation and optimization in assisted reproductive technology (ART) cycles, especially IUI, is of particular importance. It is worth noting that the use of antioxidants not only has direct effect on biological parameters of sperm such as number, motility, morphology, and agglutination rate but also can change the sperm capacitation and the rate of acrosome reaction in sperm by modifying all specific indexes of sperm fertility and ultimately affect the ability of the sperm to fertilize the ovum (19). In this regard, it has been reported that the use of antioxidant compounds in patients with oligospermia or asthenozoospermia, or those who have high levels of ROS (oxidative stress) in their samples can be a useful solution to prevent the oxidative effects of ROS on such processed spermatozoa.

The total number of spermatozoa reduced after the preparation process, because the sperm processing eliminates the dead, damaged and poorly motile spermatozoa and increases the number of appropriate sperm that are capable of fertilizing the ovum. Therefore, the reduction in the total number of spermatozoa is expected in all sperm preparation methods. In the present study, it was also observed that the sperm motility and morphology improved in the control group after processing and in the intervention groups compared to the control group, which is desirable given that the dead and damaged spermatozoa were eliminated. In the present study, as already mentioned, total sperm motility increased in all groups and the highest increase in sperm motility was observed in the NAC and myo-inositol groups.

Given the importance of the subject and the efforts to increase the quantity and quality of processed spermatozoa in ART techniques, many studies across the world have been conducted on sperm and its processing methods. The aim of this study was to increase the number of spermatozoa with normal morphological and chromosomal structures that have the potential to fertilize the ovum.

In the study of Montanino Oliva et al, the effect of myoinositol and antioxidants in men with spermatic dysfunction and metabolic disorders was shown to be greater in case of myo-inositol being used with other antioxidants (8).

As observed in our study, the motility rate increased in the NAC and myo-inositol groups. Besides, the number of TUNEL-negative cells was significantly higher in the NAC group than in the control group, and the number of TUNEL-positive cells was significantly lower in the NAC group than in the control group. In general, these effects can be due to the antioxidant effects of myo-inositol and NAC, and consequently, the prevention of adverse effects of ROS, which are consistent with the results reported by other researchers using other antioxidants and herbal compounds. In a study conducted by Barekat et al, the effect of NAC on the integrity of sperm DNA after varicocelectomy surgery was investigated and it was shown that oral administration of $200 \mathrm{mg}$ NAC could significantly increase the integrity of sperm DNA in the case group compared to the control group (20), which confirms the results of the present study. In other studies, it has been observed that myo-inositol in sperm freezing significantly increases the progressive motility and normal morphology of sperm and also reduces DNA damage. This shows that myo-inositol will prevent destructive effects that usually occur after sperm freezing, which also confirms the results of the present study. It has also been suggested that myo-inositol can be used in fertility and andrology labs and sperm banks for infertile and cancer patients who refer for sperm cryopreservation. Companies that produce sperm cryopreservation kits can use this substance in their commercially available products.

Nazm Bojnordi et al studied the effect of antioxidants on melted and frozen human sperm quality and observed that in normal individuals, the addition of vitamin $\mathrm{E}$ at concentrations of 1 and $2 \mathrm{mM}$ caused an increase in sperm motility, forward progression and survival rate, among which the increase in forward progression and survival rate was significant. This increase was also observed in individuals with oligospermia, but in general, there was no significant difference. Ascorbic acid had no significant effect on any of the sperm parameters (21). Overall, the results of the above-mentioned studies confirm the results of the present study on the positive effects of NAC and myo-inositol on maintaining the quality of processed spermatozoa. In this regard, it should be noted that during cryopreservation, semen is exposed to cold shock and osmotic pressure, 
and as a result, the amount of oxidation of the membrane increases due to the higher percentage of oxidative reactions, which ultimately leads to decrease in sperm survival and longevity. Studies have shown that fertilization rates decrease significantly after sperm cryopreservation due to its inappropriate function. Cryopreservation also leads to changes in DNA, sperm cytoskeleton, inhibition of spermegg binding, and destruction of sperm axoneme, leading to a reduction in motility. Therefore, the protection of the plasma membrane of the sperm against oxidative reactions is accomplished by antioxidants during cryopreservation. In the liquefaction stage, due to the presence of free radicals in the sperm medium, the destructive and oxidative effects of these agents on sperm are likely. In fact, antioxidants, by reducing the formation of free oxygen radicals, change the cellular conditions to preserve the sperm motility (22). In this regard, the protective effects of various antioxidants, including alpha-tocopherol, have been reported to maintain sperm quality parameters and increase its motility (23), which confirms the results of the present study.

Moreover, Papaleo et al studied the effect of myo-inositol on the quality of oocyte in intracytoplasmic sperm injection cycles with the cooperation of University of Scarborough, Canada. The researchers observed that treatment with myo-inositol and folic acid in patients with polycystic ovary syndrome (PCOS) reduced germinal vesicles and degenerated oocytes at ovum pick-up, while not affecting the total number of oocytes recovered. Therefore, this study showed the positive effect of myo-inositol on the maturation quality of the growing oocytes (24). These results are also consistent with the results of the present study on the positive effect of myo-inositol and NAC on improving the quality of processed spermatozoa. Caprio et al studied myo-inositol therapy for poor responders during IVF. In this study, 76 patients were divided into two groups, one group received myo-inositol and folic acid and the other group received only folic acid for three months. This study showed that myo-inositol had a positive effect on the quality and number of oocytes and could increase the response of the ovary to gonadotropins, and therefore, it can be used as an adjuvant therapy in poor responders during IVF (25). Taken together, these results support the use of medications such as NAC and myo-inositol due to their positive effects on sperm and ovum.

\section{Conclusion}

In the present study, NAC and myo-inositol were found to improve parameters such as sperm motility and integrity. Therefore, by using these drugs, especially NAC, sperms of higher quality can be provided to treat infertility. Therefore, it is suggested that in order to increase the efficacy of these compounds, the effects of other drug and antioxidant combinations, along with the subjects in this study, be investigated.
Conflict of Interests

The authors declare no conflict of interests.

Ethical Approval

This study was conducted in accordance with the principles of the Declaration of Helsinki and then its protocol was approved by the Ethics Committee of Shahrekord University of Medical Sciences (Approval No. IR.SKUMS.REC.1396.190).

\section{Acknowledgements}

This article was derived from the MD thesis of Naghme Samadi whose protocol was approved by the Deputy of Research and Technology of Shahrekord University of Medical Sciences (Approval No. 2618). Hereby, the financial support of the Deputy of Research and Technology of Shahrekord University and the collaboration of the Hazrat Zahra Infertility Center affiliated with the University are acknowledged.

\section{References}

1. Speroff L, Fritz M. Clinical Gynecologic Endocrinology and Infertility. 7th ed. Philadelphia: Lippincott Williams \& Wilkins; 2005.

2. Palmieri M, Papale P, Della Ragione A, Quaranta G, Russo G, Russo S. In vitro antioxidant treatment of semen samples in assisted reproductive technology: effects of myo-inositol on nemaspermic parameters. Int J Endocrinol. 2016;2016:2839041. doi: 10.1155/2016/2839041.

3. Imamovic Kumalic S, Pinter B. Review of clinical trials on effects of oral antioxidants on basic semen and other parameters in idiopathic oligoasthenoteratozoospermia. Biomed Res Int. 2014;2014:426951. doi: 10.1155/2014/426951.

4. Lombardo F, Sansone A, Romanelli F, Paoli D, Gandini L, Lenzi A. The role of antioxidant therapy in the treatment of male infertility: an overview. Asian J Androl. 2011;13(5):690-7. doi: 10.1038/aja.2010.183.

5. Hamada AJ, Montgomery B, Agarwal A. Male infertility: a critical review of pharmacologic management. Expert Opin Pharmacother. 2012;13(17):2511-31. doi: 10.1517/14656566.2012.740011.

6. Yang Y, Huang H, Feng D, Liu W, Cheng X, Ba Y, et al. [Effects. of $\mathrm{N}$-acetylcysteine on fluoride-induced endoplasmic reticulum stress in sertoli cells]. Wei Sheng Yan Jiu. 2014;43(5):805-8.

7. Agarwal A, Saleh RA. Role of oxidants in male infertility: rationale, significance, and treatment. Urol Clin North Am. 2002;29(4):817-27. doi: 10.1016/s0094-0143(02)00081-2.

8. Montanino Oliva M, Minutolo E, Lippa A, laconianni P, Vaiarelli A. Effect of myoinositol and antioxidants on sperm quality in men with metabolic syndrome. Int J Endocrinol. 2016;2016:1674950. doi: 10.1155/2016/1674950.

9. Scarselli F, Lobascio AM, Terribile M, Casciani V, Greco P, Franco G, et al. Analysis of MYO-Inositol effect on spermatozoa motility, in hyper viscous ejaculates and in patients with grades II and III varicocele. Arch Ital Urol Androl. 2016;88(4):279-83. doi: 10.4081/aiua.2016.4.279.

10. Calogero AE, Gullo G, La Vignera S, Condorelli RA, Vaiarelli A. Myoinositol improves sperm parameters and serum reproductive hormones in patients with idiopathic infertility: a prospective double-blind randomized placebo-controlled study. Andrology. 2015;3(3):491-495. doi:10.1111/andr.12025.

11. Boni R, Gualtieri R, Talevi R, Tosti E. Calcium and other ion dynamics during gamete maturation and fertilization. Theriogenology. 2007;68 Suppl 1:S156-64. doi: 10.1016/j. theriogenology.2007.05.048.

12. Colone M, Marelli G, Unfer V, Bozzuto G, Molinari A, Stringaro A. Inositol activity in oligoasthenoteratospermia--an in vitro 
study. Eur Rev Med Pharmacol Sci. 2010;14(10):891-6.

13. Fernández JL, Cajigal D, López-Fernández C, Gosálvez J. Assessing sperm DNA fragmentation with the sperm chromatin dispersion test. Methods Mol Biol. 2011;682:291-301. doi: 10.1007/978-1-60327-409-8_21.

14. Bartoov B, Berkovitz A, Eltes F, Kogosowski A, Menezo Y, Barak $Y$. Real-time fine morphology of motile human sperm cells is associated with IVF-ICSI outcome. J Androl. 2002;23(1):1-8. doi: 10.1002/j.1939-4640.2002.tb02595.x.

15. Comhaire FH, Christophe AB, Zalata AA, Dhooge WS, Mahmoud AM, Depuydt CE. The effects of combined conventional treatment, oral antioxidants and essential fatty acids on sperm biology in subfertile men. Prostaglandins Leukot Essent Fatty Acids. 2000;63(3):159-65. doi: 10.1054/ plef.2000.0174.

16. Oeda T, Henkel R, Ohmori H, Schill WB. Scavenging effect of $\mathrm{N}$-acetyl-L-cysteine against reactive oxygen species in human semen: a possible therapeutic modality for male factor infertility? Andrologia. 1997;29(3):125-31. doi: 10.1111/ j.1439-0272.1997.tb00305.x.

17. Paradiso Galatioto G, Gravina GL, Angelozzi G, Sacchetti A, Innominato PF, Pace G, et al. May antioxidant therapy improve sperm parameters of men with persistent oligospermia after retrograde embolization for varicocele? World J Urol. 2008;26(1):97-102. doi: 10.1007/s00345-007-0218-z.

18. Condorelli RA, La Vignera S, Bellanca S, Vicari E, Calogero AE. Myoinositol: does it improve sperm mitochondrial function and sperm motility? Urology. 2012;79(6):1290-5. doi: 10.1016/j. urology.2012.03.005.
19. Kumar N, Singh AK. Trends of male factor infertility, an important cause of infertility: a review of literature. J Hum Reprod Sci. 2015;8(4):191-6. doi: 10.4103/0974-1208.170370.

20. Barekat F, Tavalaee M, Deemeh MR, Bahreinian M, Azadi $\mathrm{L}$, Abbasi $\mathrm{H}$, et al. A preliminary study: $\mathrm{N}$-acetyl-L-cysteine improves semen quality following varicocelectomy. Int J Fertil Steril. 2016;10(1):120-6. doi: 10.22074/ijfs.2016.4777.

21. Nazm Bojnordi M, Movahedin M, Amanpour S, Ghasemi Hamidabadi H. Effect of antioxidants supplementation on human sperm parameters after freezing. Journal of Mazandaran University of Medical Sciences. 2008;18(63):20-7. [Persian].

22. Breininger E, Beorlegui NB, O'Flaherty CM, Beconi MT. Alphatocopherol improves biochemical and dynamic parameters in cryopreserved boar semen. Theriogenology. 2005;63(8):212635. doi: 10.1016/j.theriogenology.2004.08.016.

23. Brezezińska-Slebodzińska $E$, Slebodziński $A B$, Pietras $B$, Wieczorek G. Antioxidant effect of vitamin E and glutathione on lipid peroxidation in boar semen plasma. Biol Trace Elem Res. 1995;47(1-3):69-74. doi: 10.1007/bf02790102.

24. Papaleo E, Unfer V, Baillargeon JP, Fusi F, Occhi F, De Santis L. Myo-inositol may improve oocyte quality in intracytoplasmic sperm injection cycles. A prospective, controlled, randomized trial. Fertil Steril. 2009;91(5):1750-4. doi: 10.1016/j. fertnstert.2008.01.088.

25. Caprio F, D'Eufemia MD, Trotta C, Campitiello MR, lanniello R, Mele D, et al. Myo-inositol therapy for poor-responders during IVF: a prospective controlled observational trial. J Ovarian Res. 2015;8:37. doi: 10.1186/s13048-015-0167-x. 